\title{
Effect of magnetic field, size and donor position on the absorption coefficients related a donor within the core/shell/shell quantum dot
}

Emre Bahadir AL ( $\nabla$ emrebahadiral@hotmail.com )

Sivas Cumhuriyet Universitesi https://orcid.org/0000-0003-4435-2879

\section{Research Article}

Keywords: donor impurity, spherical quantum dot, optical absorption coeffcient, magnetic eld

Posted Date: October 5th, 2021

DOl: https://doi.org/10.21203/rs.3.rs-844746/v1

License: (c) (i) This work is licensed under a Creative Commons Attribution 4.0 International License.

Read Full License

Version of Record: A version of this preprint was published at Optical and Quantum Electronics on November 2nd, 2021. See the published version at https://doi.org/10.1007/s11082-021-03340-w. 


\title{
Effect of magnetic field, size and donor position on the absorption coefficients related a donor within the core/shell/shell quantum dot
}

\author{
E. B. $\mathrm{Al}^{\mathrm{a}}$ \\ Faculty of Science, Department of Physics, Sivas Cumhuriyet University, 58140 Sivas, Turkey
}

(Dated: August 24, 2021)

\begin{abstract}
In this study, linear, nonlinear and total optical absorption coefficients related a single shallow donor atom confined in semiconductor core/shell/shell quantum dot heterostructure are researched in detail within the compact density matrix formalism approximation. For this purpose, firstly, the energies and the wavefunctions are computed by the diagonalization method in the effective mass approach. Moreover, the effects of size modulation, donor position and magnetic field are analyzed. The numerical results indicate that the linear and nonlinear parts of the absorption coefficients related with intersubband $1 s \rightarrow 1 p$ and $1 p \rightarrow 1 d$ donor transitions undergo significant changes.
\end{abstract}

Keywords: donor impurity, spherical quantum dot, optical absorption coefficient, mag-

netic field

\section{INTRODUCTION}

It is generally accepted that the progress of electronic and opto-electronic devices depends on understanding the basic chemical and physical properties of low-dimensional structures (LDSs). In these LDSs, the geometric confinement limits the movement of charge carriers in space and it displays large changes in electrical and optical properties due to the occurrence of discrete energy distribution. Therefore, in recent years, intensive research activities have been conducted around the world on the behavior of matter at nanoscale. Although various devices related nanoscale particles have been developed, the properties of controlled nanoscale materials such as light emitting diodes, photo detectors and quantum dot (QD) single photon source are still the biggest problem of scientists.

Due to the last developments of semiconductor nanoelectronics, it has become possible to reduce dimensionality from bulk semiconductors to zero-dimensional semiconductor nanostructures (QDs). These nanostructures are very significant because their charge carrier motion is confined in all three directions, and therefore efficient control of the physical properties of these structures becomes possible. Proper adjustment of these physical properties makes QDs advantageous due to their potential application in the development of semiconductor optoelectronic devices. Therefore, some optical properties of semiconductor QDs such as dipole transition [1, 2], oscillator strength [3, 4], photoionization cross-section [5], optical absorption coefficients (OACs) [6, 7] and refractive index changes (RICs) $[3,8]$ have attracted great interest in experimental and theoretical studies in last years.

The present information on the optical properties of nanomaterials is the product of previous studies carried out by many scientists, and the optical properties of QDs have attracted much interest theoretically in last years. Conducted studies include researches on the effects of external perturbations such as electric field, magnetic field, intense laser field, pressure and temperature [9-11]. The results indicate that these perturbations allow the possibility of adjusting the value of oscillator strength and the peak resonance frequencies, thus purports an chance to change the optical response.

The electronic and optical properties in a QD are affected by the asset of impurities. Some studies [12-14] have showed that the realization of single dopant scheme in QDs is possible. This enables a broad range of potential optoelectronic devices. It is notable that due to the presence of impurity and other external factors, the computation of energies and wavefunctions is a work that takes remarkable theoretical working. Because of the complicated practical geometries, boundary conditions and effects of the surrounding environment, it is generally not feasible to find analytical solutions using familiar standard methods. To accomplish this obstacle, researchers have used different methods such as variational approximation [15], tight-binding model [16], perturbation theory [17] and diagonalization method [6].

\footnotetext{
a emrebahadiral@hotmail.com
} 
In theoretical studies on QDs, it is usual to get a spherical shape and many works have focused on the optical properties of this type of structure [3, 6, 18-20]. Thanks to the rapid development of crystal magnification procedures such as molecular beam epitaxy, metal organic chemical vapor deposition and electron lithography, and the advancement of chemical production processes, various dimensional new generation quantum nanostructures have been produced and one of which is core/shell/shell QD (CSSQD) [21, 22]. These structures consist of semiconductor materials with different band alignment. They are spatially alligned in such a way that a larger bandwidth core, acting as a substrate, is covered by smaller bandwidth spherical shells (Fig. 1). The originality of these structures is that their physical properties can be regulated in a controlled manner, resulting in changing energy levels. Therefore, it is significant to examine the electronic and optical properties of CSSQDs.

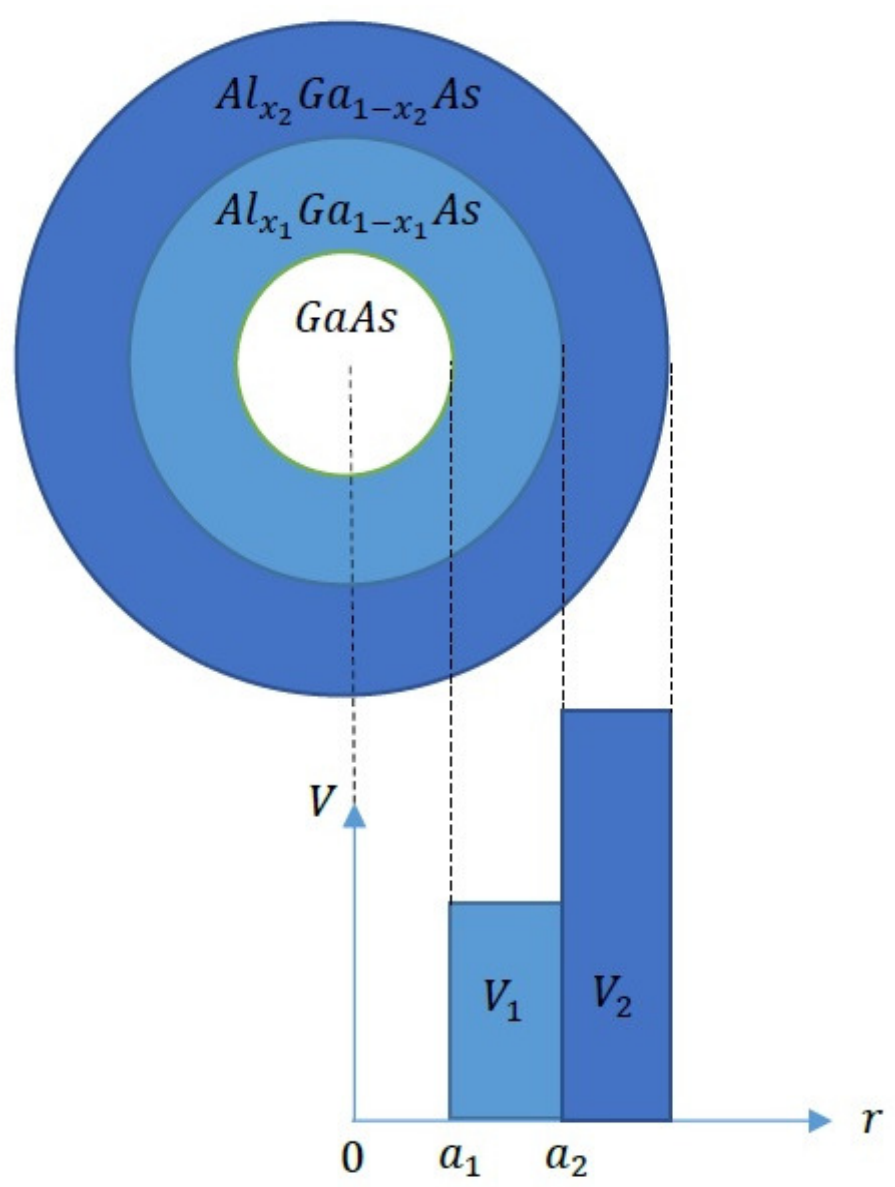

FIG. 1. Schematic presentment of the spherical CSSQD with the associated potential energies.

Some studies on OACs in spherical core/shell QDs have been previously published. The OACs and RICs related $1 s-1 p$ transition of an impurity confined in the $C d S e / Z n T e$ core/shell spherical heteronanodot were theoretically investigated by Chafai et al [23]. OACs and relative RICs in the GaAs/AlAs core/shell QD were calculated by M'zerd et al [24], considering the influences of interaction with the LO-phonon and the localization of a donor center. Feddi et al [25] researched nonlinear OACs and RICs related with the $1 s-1 p$ transition of a single impurity in $\mathrm{AlAs} / \mathrm{GaAs} / \mathrm{SiO} 2$ core/shell QDs, considering the influences of core/shell sizes, magnetic field, and dielectric mismatch. The effect of pressure on optical properties and binding energy associated with the $1 s-1 p$ intersubband transition of an impurity in an $\mathrm{AlAs} / \mathrm{GaAs}$ spherical core/shell QD was researched by Haouari et al [26]. Intersubband OACs and RICs in GaAs/AlGaAs core/shell spherical QDs were theoretically studied by Zhang et al [27] for both cases with and without on-center dopant. The electronic and optical properties of a donor in the $C d T e / Z n_{0.2} C d_{0.8} T e / Z n T e$ CSSQD have 
been discussed by Jasmine et al [28] considering the geometric confinement effect.

However, there is no study in the literature on OACs caused by $1 s \rightarrow 1 p$ and $1 p \rightarrow 1 d$ single dopant transitions in $G a A s / A l_{x_{1}} G a_{1-x_{1}} A s / A l_{x_{2}} G a_{1-x_{2}} A s$ CSSQD under the effect of a magnetic field. Therefore, in available theoretical study, linear, nonlinear and total OACs are investigated using density matrix formalism. The computation of the electronic state in the presence of both the donor impurity and the magnetic field is made by the diagonalization method. As a result of this research, it has been shown that the OACs of this structure are strictly dependent on external magnetic field, donor position and its geometric parameters such as core and shell thickness. It is hoped that this study will allow a lot elaborate specification of the donor states in the nanostructure researched.

\section{THE APPLIED THEORY}

\section{A. Electron-impurity Hamiltonian and wave function}

Since the optical behavior of nanoscale semiconductors is related to their electronic properties, the effects of varying size and external fields on the energy behavior of confined donor must first be studied.

Consider a hydrogen-like shallow donor located anywhere in the core region of a CSSQD nanocrystal with a spherically symmetrical design (Fig. 1). GaAs core material with wide band gap and radius $a_{1}$ is covered with $A l_{x_{1}} G a_{1-x_{1}} A s$ inner shell with a narrower band gap and shell thickness $T_{s}=a_{2}-a_{1}$. The outer shell consists of $A l_{x_{2}} G a_{1-x_{2}} A s$, which has a much narrower band gap and a thickness so large that quantum effects cannot be observed. The system is exposed to a magnetic field applied externally. In the effective mass approach, the Hamiltonian that describes the energy behavior of a single electron bound to the donor confined in such a system can be stated as

$$
H=\frac{1}{2 m^{*}}\left(\vec{p}+\frac{e}{c} \vec{A}\right)^{2}+V(r)+V_{C},
$$

where, the first term is the kinetic energy operator in the presence of the magnetic field, $m^{*}$ is the conduction band effective mass of the electron in the GaAs-core region (since the electron is mostly localized in the $G a A s$-core region, the effective mass of this region is used), $e$ is the charge of electron and $c$ is the light speed in free space. Also, $\vec{A}$ is the magnetic vector potential defined as $\vec{A}=\frac{1}{2} \vec{B} \times \vec{r}$ in the Coulomb gauge, where $\vec{B}$ is chosen to directed throughout the $z$-axis $(\vec{B}=B \hat{k})$.

The second term in the Eq. 1 represents the confinement potential and it is writen in the form

$$
V(r)= \begin{cases}0, & r<a_{1} \\ V_{1}, & a_{1} \leq r<a_{2} \\ V_{2}, & r \geq a_{2}\end{cases}
$$

where $V_{1,2}=0.6\left(1247 x_{1,2}\right) \mathrm{meV}[29]$ is the conduction band offset between AlGaAs and GaAs and $x_{1}\left(x_{2}\right)$ is the aluminum concentration in the inner (outer) shell.

The third term in the Eq. 1 shows the Coulomb potential energy between the donor impurity and the electron and it has the form

$$
V_{C}=-\frac{e^{2} Z}{\varepsilon\left|\vec{r}-\vec{r}_{d}\right|},
$$

where $Z=0$ and $Z=1$ correspond to the case without and with an impurity in the structure, respectively, $\varepsilon$ is the dielectric constant, $\left|\vec{r}-\vec{r}_{d}\right|$ is the electron-impurity distance, $\vec{r}$ and $\vec{r}_{d}$ are the radial locations of the electron and the ionized impurity with respect to the center of the QD, respectively. It is assumed that the impurity is placed along the $z$-axis. In terms of spherical harmonics $\frac{1}{\left|\vec{r}-\vec{r}_{d}\right|}$ term is expressed as [17]

$$
\frac{1}{\left|\vec{r}-\vec{r}_{d}\right|}=\sum_{\mu} \frac{4 \pi}{2 \mu+1} f_{\mu}(r) \sum_{\nu=-\mu}^{\mu} Y_{\mu, \nu}^{*}(\theta, \phi) Y_{\mu, \nu}^{*}\left(\theta_{d}, \phi_{d}\right),
$$

where, $\theta$ and $\phi\left(\theta_{d}\right.$ and $\left.\phi_{d}\right)$ are the polar angles of the electron (donor), and $f_{\mu}(r)$ is

$$
f_{\mu}(r)= \begin{cases}\frac{1}{r_{d}}\left(\frac{r}{r_{d}}\right)^{\mu}, & r \leq r_{d} \\ \frac{1}{r}\left(\frac{r_{d}}{r}\right)^{\mu}, & r \geq r_{d}\end{cases}
$$


At this point, to reduce numerical calculations, reduced units are used, describing $R y d=\frac{\hbar^{2}}{2 m^{*} a_{B}^{2}}$ as energy unit and $a_{B}=\frac{\hbar^{2} \varepsilon}{m^{*} e^{2}}$ as length unit and the Hamiltonian becomes

$$
H=-\nabla^{2}+V(r)-i \gamma \frac{\partial}{\partial \phi}+\frac{\gamma^{2} r^{2} \sin ^{2} \theta}{4}-2 Z \sum_{\mu} \frac{4 \pi}{2 \mu+1} f_{\mu}(r) \sum_{\nu=-\mu}^{\mu} Y_{\mu, \nu}^{*}(\theta, \phi) Y_{\mu, \nu}^{*}\left(\theta_{d}, \phi_{d}\right)
$$

where the dimensionless $\gamma=\frac{e B a_{B}^{2}}{\hbar c}$ parameter defines the magnetic field strength.

To get the energies and wavefunctions, the Schrödinger equation $H \psi_{n l m}(r, \theta, \phi)=E_{n l m} \psi_{n l m}(r, \theta, \phi)$ must be solved, where $E_{n l m}$ is the energy eigenvalue for certain quantum numbers ( $n$ : principal quantum number, $l$ : angular momentum quantum number, $m$ : magnetic momentum quantum number) and $\psi_{n l m}(r, \theta, \phi)$ is the wavefunction corresponding to this energy. The angular momentum quantum number $l=0,1,2, \ldots$ is marked by the familiar impression $s, p, d, \ldots$. The diagonalization method is used to resolve the Schrödinger equation and for this purpose, the electron wavefunctions of the infinite QD are taken as the basis function [30-32]

$$
\psi_{n l m}(r, \theta, \phi)=\sum_{j} c_{n_{j} l_{j} m} \psi_{n_{j} l_{j} m}^{(0)}(r, \theta, \phi) .
$$

where $c_{n l m}$ are the expansion coefficients (the size of the expansion is adjusted by performing a convergence test for each energy state), $\quad{ }_{n l m}^{(0)}(r, \theta, \phi)$ is the total wavefunction describing the movement of the electron without the magnetic field and the donor impurity, and the exact solutions for an electron in an infinite radial potential are [6]

$$
{ }_{n l m}^{(0)}(r, \theta, \phi)=\varphi_{n l}^{(0)}(r) Y_{l m}(\theta, \phi)
$$

where the radial wavefunction- $\varphi_{n l}^{(0)}(r)$ is given as

$$
\varphi_{n l}^{(0)}(r)=\left\{\begin{array}{ll}
N j_{l}\left(k_{n l} r\right), & r<a_{3} \\
0, & r \geq a_{3}
\end{array},\right.
$$

where $N$ is the normalization constant, $k_{n l}$ is the $n t h$ root of the spherical Bessel function- $j_{l}$ and $a_{3}\left(a_{3}>>a_{2}\right)$ is the radius of the infinite spherical QD.

\section{B. Optical absorption coefficients}

The optical transition from a first state to a latest state by a photon is commonly known as photon absorption. For any transition in LDSs, these absorptions are thought to be important for investigating new optical properties.

In a monochromatic electromagnetic field with a frequency $\omega$, the possibility of transition between states $i$ and $j$ is known as the oscillator strength $P_{i j}$ expressed by the Fermi golden rule and it is expressed in the form [26]

$$
P_{i j}=\frac{2 m^{*}}{\hbar^{2}} E_{i j}\left|M_{i j}\right|^{2}
$$

where, $E_{i j}=E_{j}-E_{i}$ is the energy difference between the first and latest states, while $M_{i j}$ is the dipole matrix element (DME) of the transition between the $i$ and $j$ states. The oscillator strength is highly dependent on the overlap of wavefunctions and the energy difference between states and thus it gives an idea of the dominant color of the emitted light.

OACs can be acquired by density matrix approximation and linear and nonlinear sensitivities for a two-level atomic system are given as

$$
\chi_{\omega}^{(1)}(\omega)=\frac{\sigma_{s}\left|M_{i j}\right|^{2}}{\varepsilon_{0}\left(E_{i j}-\hbar \omega-i \hbar \Gamma_{i j}\right)}
$$

and

$$
\chi_{\omega}^{(3)}(\omega)=\frac{\sigma_{s}\left|M_{i j}\right|^{2}|\tilde{E}|^{2}}{\varepsilon_{0}\left(E_{i j}-\hbar \omega-i \hbar \Gamma_{i j}\right)}\left[\frac{4\left|M_{i j}\right|^{4}}{\left(E_{i j}-\hbar \omega\right)^{2}+\left(\hbar \Gamma_{i j}\right)^{2}}-\frac{\left|M_{j j}-M_{i i}\right|^{2}}{\left(E_{i j}-i \hbar \Gamma_{i j}\right)\left(E_{i j}-\hbar \omega-i \hbar \Gamma_{i j}\right)}\right]
$$

respectively, where, $\sigma_{s}$ is the electron density in the QD, $\varepsilon_{0}$ is the dielectric constant of the free space, $\hbar \omega$ is the incident photon energy, $\hbar \Gamma_{i j}$ is Lorentzian line width, $\Gamma_{i j}=1 / T_{i j}$ is the non-diagonal damping term known as the relaxation ratio between the first and latest states and it is described as the reverse of the relaxation time $\left(T_{i j}\right)$. 
Sensitivity $\chi(\omega)$ is associated with the OAC in the form

$$
\alpha(\omega)=\omega \varepsilon_{0} \sqrt{\frac{\mu}{\varepsilon_{r}}} \operatorname{Im}[\chi(\omega)]
$$

where, $\mu$ indicates the magnetic susceptibility and $\varepsilon_{r}$ is the dielectric permeability of the material. In this way, the linear, nonlinear and total intersubband OACs are theoretically formulated as

$$
\begin{gathered}
\alpha^{(1)}(\omega)=\sqrt{\frac{\mu}{\varepsilon_{r}}} \frac{\sigma_{s} \Gamma_{i j}}{\left(E_{i j}-\hbar \omega\right)^{2}+\left(\hbar \Gamma_{i j}\right)^{2}} \hbar \omega\left|M_{i j}\right|^{2}, \\
\alpha^{(3)}(\omega, I)=-\sqrt{\frac{\mu}{\varepsilon_{r}}}\left(\frac{I}{2 n_{r} \varepsilon_{0} c}\right) \frac{\sigma_{s} \Gamma_{i j} \hbar \omega\left|M_{i j}\right|^{2}}{\left[\left(E_{i j}-\hbar \omega\right)^{2}+\left(\hbar \Gamma_{i j}\right)^{2}\right]^{2}}\left[4\left|M_{i j}\right|^{2}-\frac{\left|M_{j j}-M_{i i}\right|^{2}\left[3 E_{i j}^{2}-4 E_{i j} \hbar \omega+\hbar^{2}\left(\omega^{2}-\Gamma_{i j}^{2}\right)\right]}{E_{i j}^{2}+\left(\hbar \Gamma_{i j}\right)^{2}}(15)\right.
\end{gathered}
$$

and

$$
\alpha(\omega, I)=\alpha^{(1)}(\omega)+\alpha^{(3)}(\omega, I)
$$

respectively, where $I=2 \varepsilon_{0} n_{r} c|\tilde{E}|^{2}$ is the intensity of the linearly polarized electromagnetic field, $\tilde{E}$ is the amplitude of this electromagnetic field and $n_{r}$ is the relative refractive index of the structure.

Near the resonance, the second term of the parenthesis in Eq. 15 causes little effect in nonlinear OAC due to the small value of $M_{j j}-M_{i i}$ and thus it can be neglacted. In this case, the resonance condition is $\hbar \omega=\sqrt{E_{i j}^{2}+\left(\hbar \Gamma_{i j}\right)^{2}}$ for linear OAC and $\hbar \omega=\frac{1}{3}\left(E_{i j}+\sqrt{4 E_{i j}^{2}+3\left(\hbar \Gamma_{i j}\right)^{2}}\right)$ for nonlinear OAC. Thus, the maximum values of linear and nonlinear OACs are given in the forms

$$
\alpha_{\max }^{(1)}(\omega)=\sqrt{\frac{\mu}{\varepsilon_{r}}} \frac{\sigma_{s} \Gamma_{i j}}{\left(E_{i j}-\hbar \omega\right)^{2}+\left(\hbar \Gamma_{i j}\right)^{2}} \Omega_{1}
$$

and

$$
\alpha_{\max }^{(3)}(\omega, I)=-\sqrt{\frac{\mu}{\varepsilon_{r}}}\left(\frac{2 I}{n_{r} \varepsilon_{0} c}\right) \frac{\sigma_{s} \Gamma_{i j}}{\left[\left(E_{i j}-\hbar \omega\right)^{2}+\left(\hbar \Gamma_{i j}\right)^{2}\right]^{2}} \Omega_{2}
$$

respectively, where, $\Omega_{1}=e^{-2} \sqrt{E_{i j}^{2}+\left(\hbar \Gamma_{i j}\right)^{2}}\left|M_{i j}\right|^{2}$ is the reduced dimensionless oscillator strength and $\Omega_{2}=$ $\frac{e^{-4}}{3}\left(E_{i j}+\sqrt{4 E_{i j}^{2}+3\left(\hbar \Gamma_{i j}\right)^{2}}\right)\left|M_{i j}\right|^{4}$.

Assuming that electromagnetic radiation is linearly polarized throughout the $z$-axis, the DME is defined in a single electron system by

$$
M_{i j}=\left\langle\psi_{i}|\operatorname{er} \cos \theta| \psi_{j}\right\rangle
$$

where, $\psi_{i}$ and $\psi_{j}$ are wavefunctions of the first and latest states, respectively. In order for the DME to be different from zero, the selection rules $\Delta l= \pm 1$ and $\Delta m=0, \pm 1$ must be provided. In this study, the intersubband transitions between only $m=0$ states in the CSSQD will be discussed.

\section{NUMERICAL RESULTS AND DISCUSSION}

In this section, the effects of magnetic field, geometric confinement and donor position change on OACs will be discussed according to the spherical CSSQD nanostructure model outlined above. The values of the material input parameters taken into account in the computation are presented as follows: $\varepsilon=13.18$ (since the electron is mostly localized in the GaAs-core region, the dielectric constant of this region is used), $m^{*}=0.067 m_{0}($ where $m_{0}=9.10956 \times 10^{-31} \mathrm{~kg}$ is the free electron mass), $I=400 \mathrm{MW} / \mathrm{m}^{2}, \sigma_{s}=1 \times 10^{23} \mathrm{~m}^{-3}, T_{i j}=0.14 \mathrm{ps}, n_{r}=3.2$, $a_{B}=10.42 \mathrm{~nm}, \mathrm{Ryd}=5.23 \mathrm{meV}$.

In Fig. 2, the change of linear, nonlinear and total OACs related to $1 s \rightarrow 1 p$ and $1 p \rightarrow 1 d$ transitions for the central donor atom in the absence of a magnetic field are plotted as function of the incident photon energy considering various core/shell sizes. As can be seen from the Eqs. 17 and 18, OACs reach the maximum when $\hbar \omega \approx E_{i j}$, and the peak amplitudes of linear and nonlinear OACs are proportional to $\Omega_{1}$ and $\Omega_{2}$, respectively. In Fig. 2 (a), the transition 
energy (TE) decreases as a result of closing the energy difference between the $1 s$ and $1 p$ states due to the increase in the size of the core material, while the related wave functions more overlap. The competitive influence between these two factors induces the peak amplitude of linear OAC to be senseless to change in core size. However, the fourth power of the DME in the nonlinear OAC is more dominant and thus the peak magnitude of the nonlinear OAC is increased. In addition, it can be noticed that the nonlinear OAC has a large effect on the total OAC, thus the peak magnitude of the total OAC is decreased. It is quite clear that the increase in core size improves the peak magnitudes of both linear and nonlinear OACs associated with the $1 p \rightarrow 1 d$ transition. Because the growing core size pushes down the tunneling effect and hence the electron in the $1 d$ state is chiefly confined in the core region, the wave functions of the $1 p$ and $1 d$ states overlap, thus the peak magnitudes of the OACs increase. However, the peak amplitudes of OACs depend on the TE as well as the overlap of the wavefunctions. In contrast to the increasing overlap of wavefunctions, the TE incurs a decrease with growing core size. Here, it can be said that the DME is more dominant than the TE. Other than this, as the nonlinear OAC is affected more by the core size than the linear one, the peak magnitude of the total OAC is decreased. Also, the peak positions of the OACs show a redshift with the increase in core size. This is because the energy difference between the $1 p$ and $1 d$ states in the large core size is smaller than the energy difference in the small core size. All these changes correspond exactly to the values in Table I.

In Fig. 2 (b), no change is observed in the peak magnitudes and positions of the OACs related to the $1 s \rightarrow 1 p$ transition when the shell thickness increases. Because the change in shell thickness is not affect the probability distributions and energy levels of $1 s$ and $1 p$ states. As can be seen from Table I, there is no significant change in the TE nor in the $\Omega_{1}$ and $\Omega_{2}$ factors. The peak amplitudes of the OACs related to the $1 p \rightarrow 1 d$ transition decrease and the peak positions show a redshift with growing shell thickness. Because, although the probability density of the $1 p$ state does not change with the growing in the shell thickness, the probability density of the $1 d$ state is localized in a wider region within the structure, so the DME is decreased. As a combined result of both the decreasing of the DME and the redshift of the TE, the $\Omega_{1}$ and $\Omega_{2}$ multipliers and consequently the peak amplitudes of the OACs are decreased. 

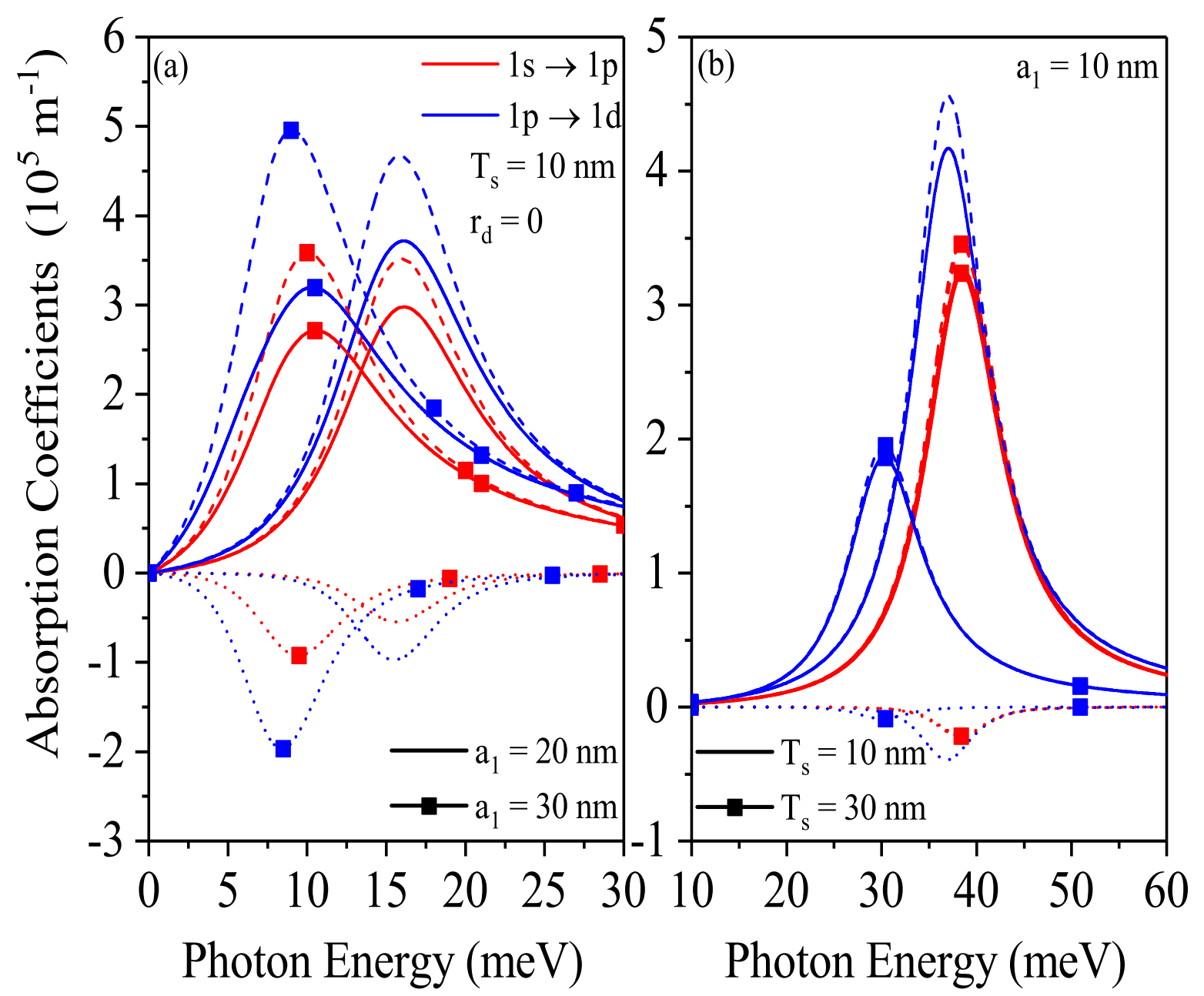

FIG. 2. Linear (dashed), nonlinear (dotted) and total (solid) OACs related to $1 s \rightarrow 1 p$ (red) and $1 p \rightarrow 1 d$ (blue) on-center donor transitions as a function of incident photon energy in the spherical $G a A s / A l_{0.1} G a_{0.9} A s / A l_{0.4} G a_{0.6} A s$ CSSQD, for different core/shell sizes without magnetic field.

To examine the influence of the position of donor atom in the core region, OACs are presented in Fig. 3 against the incident photon energy for different positions of the impurity without a magnetic field. The peak magnitude of the linear $\mathrm{OAC}$ for the $1 s \rightarrow 1 p$ transition is greatest when the donor atom is at the center of QD, but it remains constant outside the center. On the other hand, the peak magnitude of the nonlinear OAC is greatest when the impurity is at the core/shell boundary, but it remains constant at other positions. However, the peak locations of the OACs show a redshift as the impurity draws away from the dot center, due to the convergence of $1 s$ and $1 p$ energy levels. As can be understood from these changes, although the DME increases at the core/shell boundary, the peak magnitude of the linear OAC does not vary due to competition between the DME and the TE. However, the peak magnitude of the nonlinear OAC rises due to the fact that it is proportional to the fourth power of the DME. Since the peak amplitudes of the nonlinear OAC are smaller than the linear ones, the peak amplitudes of the total OAC show an equivalent variation with the linear ones except for a small decrease in amplitude. The same is true for the $1 p \rightarrow 1 d$ transition. However, the peak locations of the OACs for the $1 p \rightarrow 1 d$ transition is shifted first slightly blue and then red as the donor atom draws away from the QD center. This is because of the fact that the energy difference between the $1 p$ and $1 d$ levels before rises and after that decreases as the donor atom draws away from the QD center. While the peak magnitude of the linear OAC is not affected by the impurity location, the peak amplitude of the nonlinear OAC is increased at the core/shell boundary, so it can be said that the DME is larger at the core/shell boundary. All 
TABLE I. In the absence of the magnetic field, the TEs and the values of some factors $\left(\Omega_{1}\right.$ and $\left.\Omega_{2}\right)$ for different core/shell sizes. The values are for $x_{1}=0.1, x_{2}=0.4$ and $r_{d}=0$.

\begin{tabular}{c|c|c|c|c} 
& \multicolumn{2}{|c|}{ Sizes $(n m)$} & $1 s \rightarrow 1 p$ & $1 p \rightarrow 1 d$ \\
\hline \multirow{3}{*}{$E_{i j}(\mathrm{meV})$} & \multirow{2}{*}{$T_{s}=10$} & $a_{1}=20$ & 15.25 & 15.13 \\
& & $a_{1}=30$ & 8.85 & 7.74 \\
\cline { 2 - 5 } & \multirow{2}{*}{$a_{1}=10$} & $T_{s}=10$ & 38.33 & 36.70 \\
& & $T_{s}=30$ & 38.10 & 29.91 \\
\hline \multirow{3}{*}{$\Omega_{1}\left(\mathrm{meVnm}^{2}\right)$} & \multirow{2}{*}{$T_{s}=10$} & $a_{1}=20$ & 570 & 757 \\
\cline { 2 - 5 } & \multirow{2}{*}{$a_{1}=10$} & $a_{1}=30$ & 568 & 778 \\
\hline \multirow{3}{*}{$\Omega_{2}\left(\mathrm{meVnm}^{4}\right)$} & $T_{s}=30$ & 568 & 747 \\
\cline { 3 - 5 } & \multirow{2}{*}{$T_{s}=10$} & $a_{1}=20$ & 20957 & 37273 \\
\cline { 2 - 5 } & $a_{1}=10$ & $a_{1}=30$ & 34915 & 73968 \\
\hline & & $T_{s}=30$ & 8392 & 15162 \\
& & & & 3383
\end{tabular}

changes described are consistent with the values in Table II.

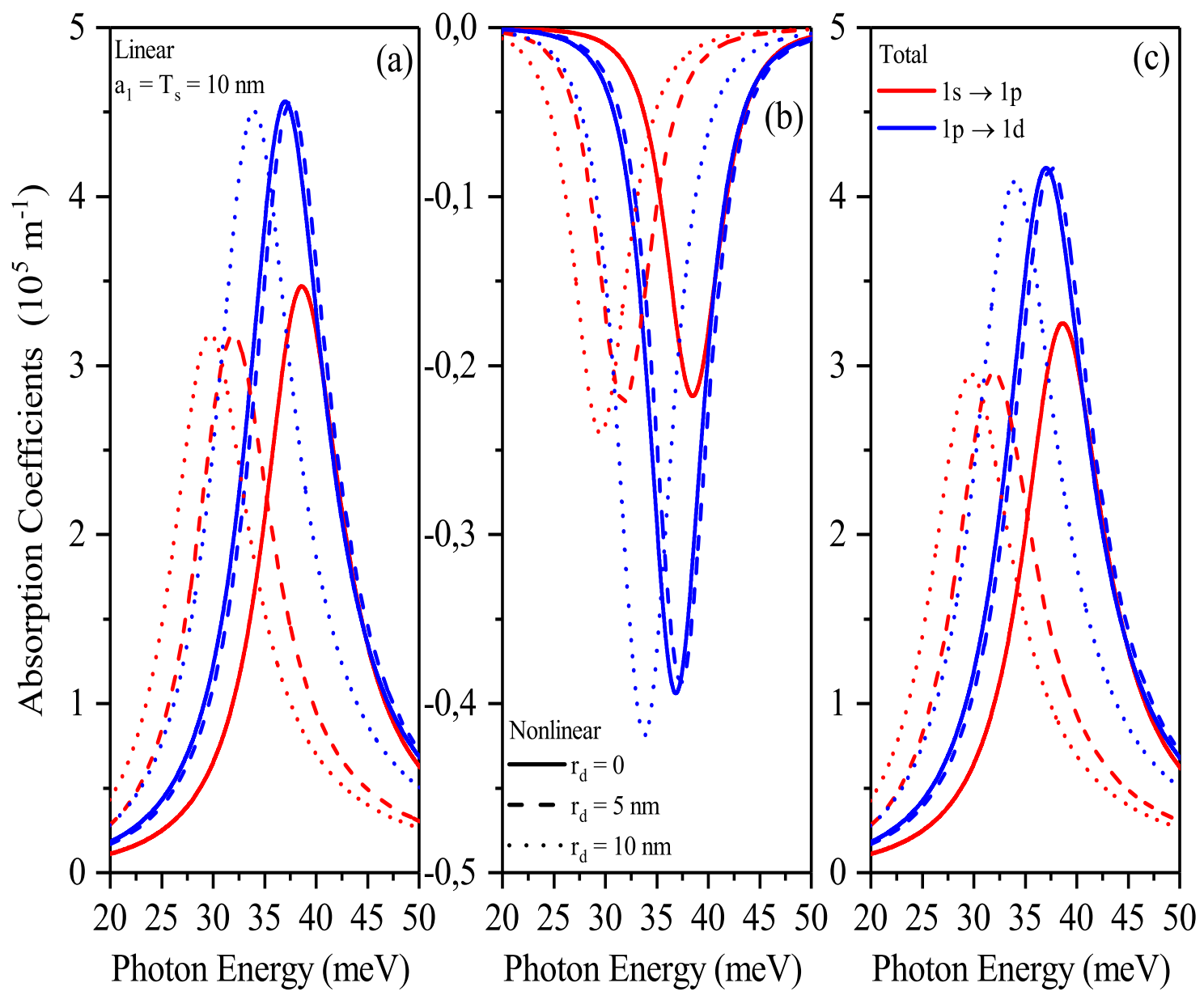


FIG. 3. Linear (a), nonlinear (b) and total (c) OACs against incident photon energy for $a_{1}=T_{s}=10 \mathrm{~nm}$ and three impurity position values without magnetic field. The values are for $x_{1}=0.1, x_{2}=0.4$ and $a_{1}=T_{s}=10 \mathrm{~nm}$.

TABLE II. In the absence of the magnetic field, the TEs and the values of some factors $\left(\Omega_{1}\right.$ and $\left.\Omega_{2}\right)$ for different donor positions. The values are for $x_{1}=0.1, x_{2}=0.4$ and $a_{1}=T_{s}=10 \mathrm{~nm}$.

\begin{tabular}{c|c|c|c} 
& $r_{d}(\mathrm{~nm})$ & $1 s \rightarrow 1 p$ & $1 p \rightarrow 1 d$ \\
\hline \multirow{3}{*}{$E_{i j}(\mathrm{meV})$} & 0 & 38.33 & 36.70 \\
& 5 & 31.59 & 37.19 \\
& 10 & 29.32 & 33.61 \\
\hline \multirow{3}{*}{$\Omega_{1}\left(\mathrm{meVnm}{ }^{2}\right)$} & 0 & 568 & 747 \\
& 5 & 520 & 746 \\
& 10 & 521 & 737 \\
\hline \multirow{3}{*}{$\Omega_{2}\left(\mathrm{meVnm}^{4}\right)$} & 0 & 8392 & 15162 \\
& 5 & 8533 & 14927 \\
& 10 & 9226 & 16131
\end{tabular}

To understand the influence of the magnetic field, linear, nonlinear and total contributions to the OACs as a function of the incident photon energy in the absence and presence of the magnetic field for different core/shell sizes are drawn in Fig. 4. It is supposed that the donor atom is placed at the QD center and the entire structure is under the effect of the magnetic field. Regardless of the core/shell sizes, it can be understood that the peak locations of the OACs undergo a blueshift under the influence of the magnetic field. This is because the magnetic field creates an additional spatial confinement. That is, with the effect of the magnetic field, the spatial distribution of the electron wavefunction becomes weaker and the cyclotron radius decreases. As a result, the TE rises as given in Table III and the OACs shift to blue. For the core/shell sizes in the left column given in Fig. 4, it should be noted that with the influence of the magnetic field, there is a significant increment in the peak amplitudes of the OACs associated with the $1 p \rightarrow 1 d$ transition. This is because both the TE and the DME increase with the effect of the magnetic field. Also, since the peak magnitude of the nonlinear OAC is small compared to the linear one, the peak magnitude of the total OAC is nearly the same as the linear one. Other than this, the small increase in TE for the $1 s \rightarrow 1 p$ transition is balanced by the small decrement in the DME and consequently the OAC peak amplitudes are not change. For the core/shell sizes in the right column given in Fig. 4, with the effect of the magnetic field, the peak magnitude of the linear OAC associated with the $1 p \rightarrow 1 d$ transition increases, while the peak magnitude of the nonlinear OAC decreases. This case can be explained as follows: As emphasized before, linear and nonlinear OACs are related to the factors $\Omega_{1}$ and $\Omega_{2}$ in the Eqs. 17 and 18, respectively. These factors depend on the magnetic field as they are associated with the DME and the TE. Therefore, it can be predicted that there is a rivalry between the DME and the TE. As the TE rises with the effect of the magnetic field, the DME declines. While the TE is dominant in the amplitude change of the linear OAC, the DME is dominant in the amplitude change of the nonlinear OAC because of the fourth power of the DME. Also, numerical calculations show that the magnetic field causes a little change in the peak magnitude of nonlinear OAC compared to linear one. For this reason, the magnitude of the total OAC is dominated by the change in the linear OAC. On the other hand, for the $1 s \rightarrow 1 p$ transition, the decrement in the DME is more dominant under the effect of the magnetic field and as a result, the peak magnitudes of the OACs decline. Since the decrease in the peak magnitude of the linear OAC is greater than the decrease in the peak magnitude of the nonlinear OAC, the peak magnitude of the total OAC is also decreased. All results are in numbness with the values in Table III. 

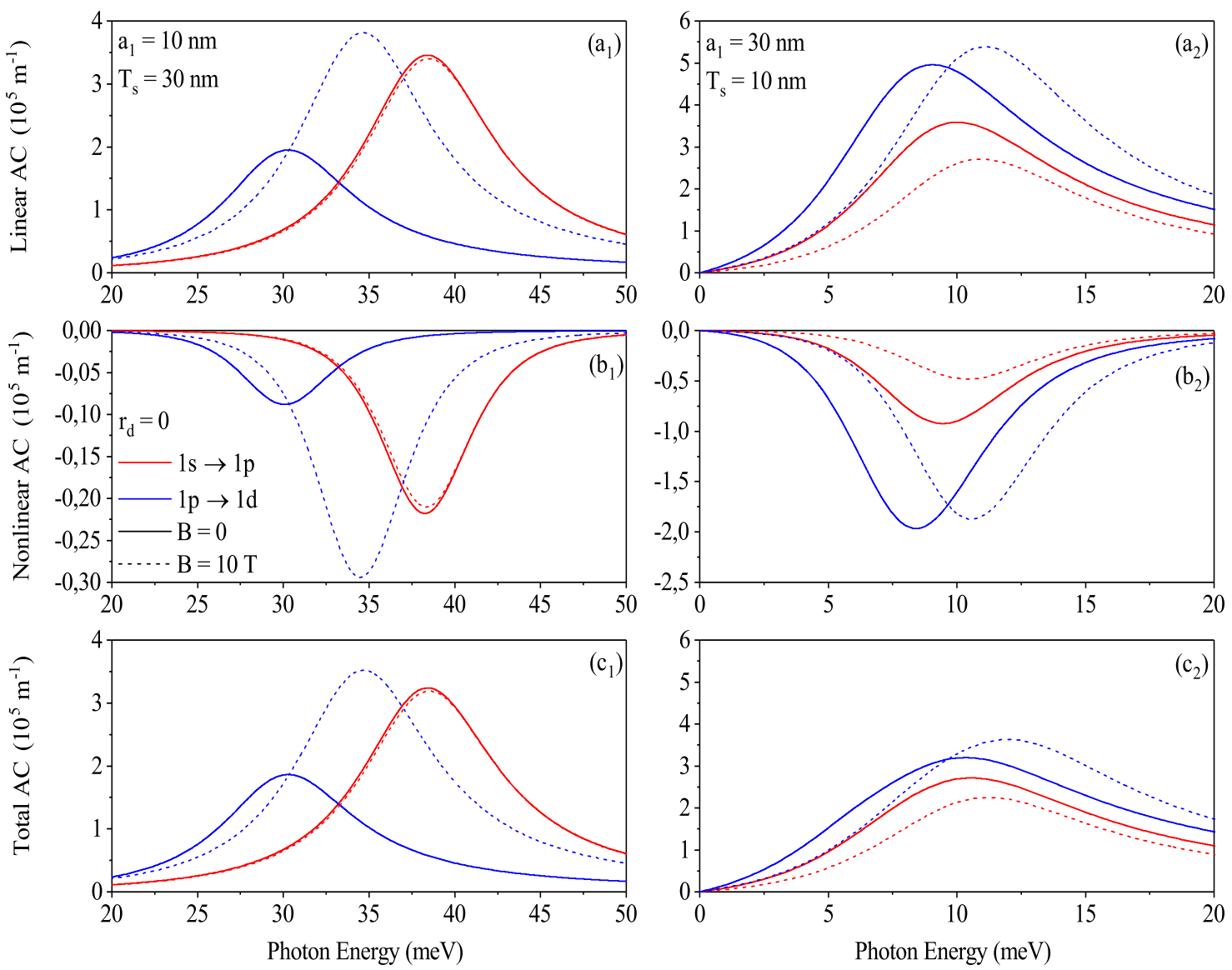

FIG. 4. Linear, nonlinear and total OACs associated with $1 s \rightarrow 1 p$ (red) and $1 p \rightarrow 1 d$ (blue) on-center impurity transitions in the $G a A s / A l_{0.1} G a_{0.9} A s / A l_{0.4} G a_{0.6} A s$ CSSQD as a function of incident photon energy in the absence (solid) and presence (dashed) of the magnetic field for several core/shell sizes.

Finally, OACs as a function of photon energy for several $x_{1}$ concentrations are introduced in Fig. 5 in the absence and presence of the magnetic field. The figure shows that increasing $x_{1}$ concentration shifts the peak locations of OACs to blue. This is because the TE increases with increasing $x_{1}$ concentration because of the quantum size effect. Besides, it has been found that the peak amplitudes of linear OACs are virtually unchanged with increasing $x_{1}$ concentration. This is because with increasing $x_{1}$ concentration, as the TE increases, the overlap of the wavefunctions of the first and latest states decreases. The peak amplitudes of the nonlinear OAC show a small decrease due to the increasing $x_{1}$ concentration. This is because the structure becomes more symmetrical as a deeper confinement potential is created with increasing $x_{1}$ concentration. On the other hand, the influence of the magnetic field is very small due to the small core/shell sizes. Note that the effect of the magnetic field is most evident for the $1 p \rightarrow 1 d$ transition for concentration $x_{1}=0.1$. Because the energy of the $1 d$ state is greater than the depth of the confinement potential for the concentration $x_{1}=0.1$, thus it is more influenced by the magnetic field as it is localized in a larger region. All these explanations can be understood by looking at the values in Table IV. 
TABLE III. In the absence and presence of the magnetic field, the TEs and the values of some factors $\left(\Omega_{1}\right.$ and $\left.\Omega_{2}\right)$ for different core/shell sizes. The values are for $x_{1}=0.1, x_{2}=0.4$ and $r_{d}=0$.

\begin{tabular}{|c|c|c|c|c|}
\hline & Sizes $(n m)$ & $B(T)$ & $1 s \rightarrow 1 p$ & $1 p \rightarrow 1 d$ \\
\hline \multirow{3}{*}{$E_{i j}(m e V)$} & \multirow{2}{*}{$a_{1}=10, T_{s}=30$} & 0 & 38.10 & 29.91 \\
\hline & & 10 & 38.19 & 34.32 \\
\hline & \multirow{2}{*}{$a_{1}=30, T_{s}=10$} & 0 & 8.85 & 7.74 \\
\hline \multirow{4}{*}{$\Omega_{1}\left(m e V n m^{2}\right)$} & & 10 & 9.81 & 10.04 \\
\hline & \multirow{2}{*}{$a_{1}=10, T_{s}=30$} & 0 & 566 & 319 \\
\hline & & 10 & 557 & 624 \\
\hline & $a_{1}=30, T_{s}=10$ & $\begin{array}{c}0 \\
10\end{array}$ & $\begin{array}{l}568 \\
432\end{array}$ & $\begin{array}{l}778 \\
850\end{array}$ \\
\hline \multirow{3}{*}{$\Omega_{2}\left(m e V n m^{4}\right)$} & \multirow{2}{*}{$a_{1}=10, T_{s}=30$} & 0 & 8383 & 3386 \\
\hline & & 10 & 8095 & 11316 \\
\hline & $a_{1}=30, T_{s}=10$ & $\begin{array}{c}0 \\
10\end{array}$ & $\begin{array}{l}34915 \\
18271\end{array}$ & $\begin{array}{l}73968 \\
71095\end{array}$ \\
\hline
\end{tabular}
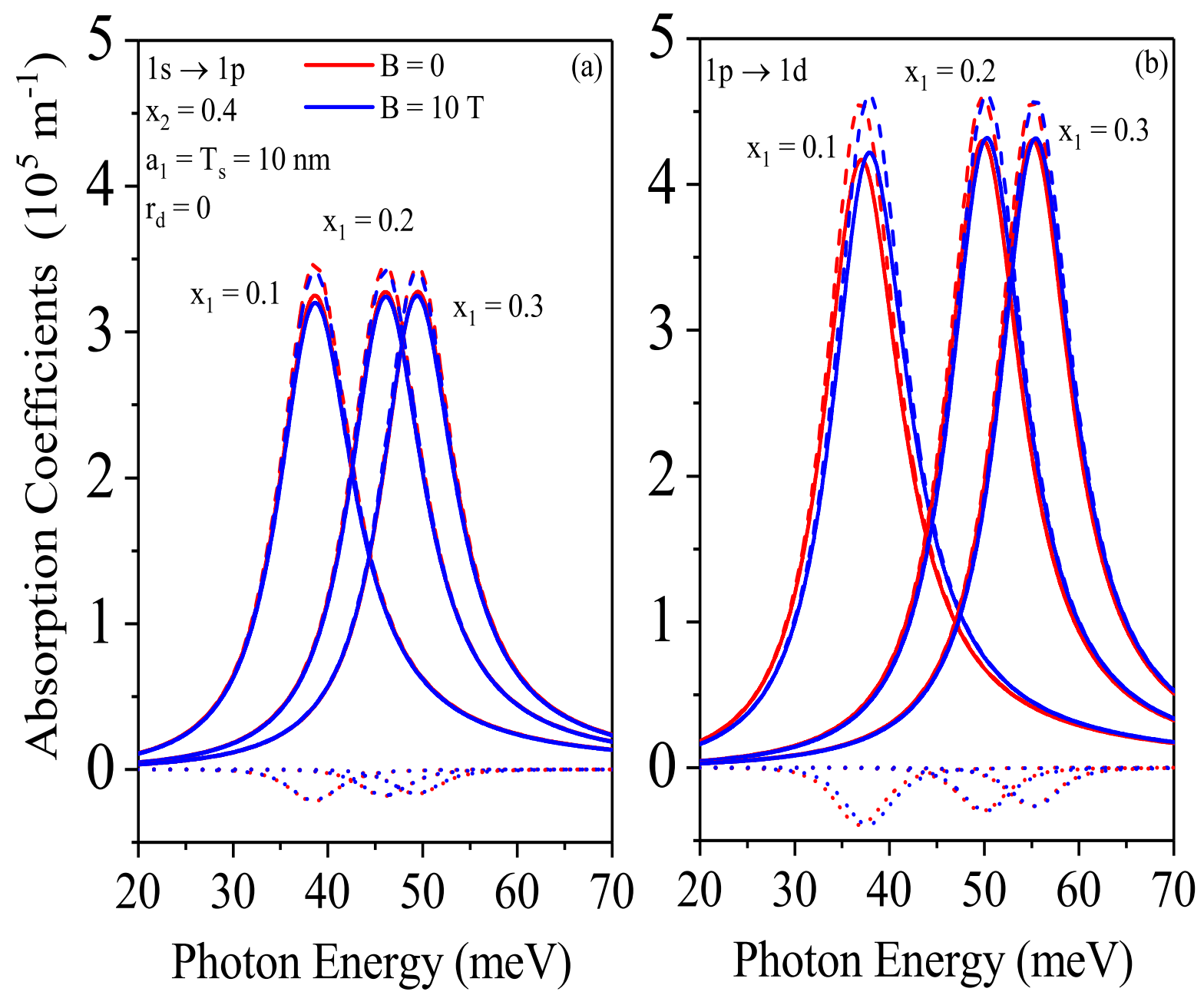

FIG. 5. Linear (dashed lines), nonlinear (dotted lines) and total (solid lines) OACs in the absence (red) and presence (blue) of the magnetic field as a function of photon energy for various $x_{1}$ concentrations: a) $1 s \rightarrow 1 p$, b) $1 p \rightarrow 1 d$. 
TABLE IV. In the absence and presence of the magnetic field, the TEs and the values of some factors $\left(\Omega_{1}\right.$ and $\left.\Omega_{2}\right)$ for different $x_{1}$ concentrations. The values are for $r_{d}=0, x_{2}=0.4$ and $a_{1}=T_{s}=10 \mathrm{~nm}$.

\begin{tabular}{|c|c|c|c|c|}
\hline & $B(T)$ & $x_{1} \mid$ & $1 s \rightarrow 1 p$ & $1 p \rightarrow 1 d$ \\
\hline \multirow{6}{*}{$E_{i j}(m e V)$} & \multirow{3}{*}{0} & 0.1 & 38.33 & 36.70 \\
\hline & & 0.2 & 45.82 & 49.63 \\
\hline & & 0.3 & 49.25 & 54.89 \\
\hline & \multirow{3}{*}{10} & 0.1 & 38.37 & 37.58 \\
\hline & & 0.2 & 45.76 & 50.04 \\
\hline & & 0.3 & 49.18 & 55.21 \\
\hline \multirow{6}{*}{$\Omega_{1}\left(m e V n m^{2}\right)$} & \multirow{3}{*}{0} & 0.1 & 568 & 747 \\
\hline & & 0.2 & 566 & 753 \\
\hline & & 0.3 & 564 & 749 \\
\hline & \multirow{3}{*}{10} & 0.1 & 558 & 755 \\
\hline & & 0.2 & 560 & 756 \\
\hline & & 0.3 & 559 & 751 \\
\hline \multirow{6}{*}{$\Omega_{2}\left(m e V n m^{4}\right)$} & \multirow{3}{*}{0} & 0.1 & 8392 & 15162 \\
\hline & & 0.2 & 6985 & 11417 \\
\hline & & 0.3 & 6453 & 10199 \\
\hline & \multirow{3}{*}{10} & 0.1 & 8105 & 15121 \\
\hline & & 0.2 & 6850 & 11407 \\
\hline & & 0.3 & 6353 & 10199 \\
\hline
\end{tabular}

\section{CONCLUSIONS}

In this theoretical study, the linear, nonlinear and total OACs of a single impurity atom confined in a spherical type CSSQD are extensively investigated by considering the effects of core/shell sizes, donor position and magnetic field. In this context, energies and wavefunctions are acquired by diagonalization method. The results obtained show the following: The peak locations of OACs are shifted to red with increasing core/shell sizes. Peak amplitudes of linear, nonlinear and total OACs related to $1 s \rightarrow 1 p$ and $1 p \rightarrow 1 d$ transitions give different responses to increased core/shell sizes due to the reasons explained above. The peak positions of the OACs related to the $1 s \rightarrow 1 p$ transition shifts clearly red as the donor atom shifts from the center of QD to the core/shell boundary, while the peak magnitudes of the linear and nonlinear OACs give maximum values at different donor positions. The peak locations and amplitudes of the OACs related to the $1 p \rightarrow 1 d$ transition give quite complex responses to the change in donor position. For the core/shell sizes examined, the magnetic field shifts the peak locations of the OACs to blue. In addition, the magnetic field mostly decreases the peak amplitudes of the OACs. In the absence and presence of the magnetic field, the increase of the $x_{1}$ concentration has no effect on the peak magnitudes of the OACs, while it shifts the peak positions clearly to blue.

As a result, it is concluded that the absorption and resonance TEs of the CSSQD can be easily controlled by setting the core/shell sizes, donor position and magnetic field. Besides, it has been found that nonlinear OAC is significant and it should be considered in the study of optical properties of donor atom in the CSSQD. Furthermore, the theoretical results obtained here may contribute to experimental works and ensure a kind of approximate forming for realistic applications such as optoelectronic devices and optical communications.

\section{Acknowledgments}

This work is supported by the Scientific Research Project Fund of Sivas Cumhuriyet University under the project number F-2021-641.

Competing interests statement

The author does not have any financial and non-financial competing interests statement.

\section{Data availability statement}


All data, figures and the program are available. The corresponding author will provide all files upon request.

\section{REFERENCES}

[1] K. Hasanirokh, A. Asgari and M. M. Rokhi, Theoretical study on nonlinear optical properties of CdS/ZnS spherical quantum dots, Optik 188, 99-103 (2019).

[2] T. A. Safeera and E. I. Anila, Synthesis and characterization of ZnGa2O4:Eu3+ nanophosphor by wet chemical method, Scripta Mater. 143, 94-97 (2018).

[3] Y. Naimi, Comment on "Magnetic field effects on oscillator strength, dipole polarizability and refractive index changes in spherical quantum dot", Chem. Phys. Lett. 767, 138380 (2021).

[4] M. A. S. Sakr, S. A. A. Gawad, S. A. El-Daly, M. T. H. A. Kana and El-Zeiny M. Ebeid, Photophysical and TDDFT investigation for (E, E)-2, 5-bis [2-(4-(dimethylamino)phenyl) ethenyl]pyrazine (BDPEP) laser dye in restricted matrices, J. Mol. Struct. 1217, 128403 (2020).

[5] V. Holovatsky, M. Chubrey and O. Voitsekhivska, Effect of electric field on photoionisation cross-section of impurity in multilayered quantum dot, Superlattice Microst. 145, 106642 (2020).

[6] E. B. Al, E. Kasapoglu, H. Sari and I. Sökmen, Optical properties of spherical quantum dot in the presence of donor impurity under the magnetic field, Physica B 613, 412874 (2021).

[7] S. Mgidlana, P. Şen and T. Nyokong, Direct nonlinear optical absorption measurements of asymmetrical zinc(II) phthalocyanine when covalently linked to semiconductor quantum dots, J. Mol. Struct. 1220, 128729 (2020).

[8] D. Yu, Z. Yu, Y. Zhang, Y. Chang and D. Yu, Cation-exchange synthesis and measurement of PbS quantum dots with high nonlinear optical properties, Optik 210, 164509 (2020).

[9] T. A. Sargsian, M. A. Mkrtchyan, H. A. Sarkisyan and D. B. Hayrapetyan, Effects of external electric and magnetic fields on the linear and nonlinear optical properties of InAs cylindrical quantum dot with modified Pöschl-Teller and Morse confinement potentials, Physica E 126, 114440 (2021).

[10] E. Kasapoglu, C. A. Duque, M. E. Mora-Ramos and I. Sökmen, The effects of the intense laser field on the nonlinear optical properties of a cylindrical Ga1-xAlxAs/GaAs quantum dot under applied electric field, Physica B 474, 15-20 (2015).

[11] L. Bouzaiene, H. Alamri, L. Sfaxi and H. Maaref, Simultaneous effects of hydrostatic pressure, temperature and electric field on optical absorption in InAs/GaAs lens shape quantum dot, J. Alloy. Comp. 655, 172-177 (2016).

[12] D. Mocatta, G. Cohen, J. Schattner, O. Millo, E. Rabani and U. Banin, Heavily doped semiconductor nanocrystal quantum dots, Science 332, 77-81 (2011).

[13] P.M. Koenraad and M.E. Flatté, Single dopants in semiconductors, Nat. Mater. 10, 91-100 (2011).

[14] D. Moraru, A. Udhiarto, M. Anwar, R. Nowak, R. Jablonski, E. Hamid, J.C. Tarido, T. Mizuno and M. Tabe, Atom devices based on single dopants in silicon nanostructures, Nanoscale Res. Lett. 6, 479-487 (2011).

[15] L. Shi, Zu-Wei Yan and Mei-Wen Meng, Binding energy and photoionization cross section of hydrogenic impurities in elliptic cylindrical core/shell quantum dots under a non-axial electric field, Superlattice Microst. 150, 106818 (2021).

[16] J. Pérez-Conde and A. K. Bhattacharjee, Electronic structure and impurity states in GaN quantum dots, Solid State Comm. 135, 496-499 (2005).

[17] Y. Yakar, B. Çakir and A. Özmen, Off-center hydrogenic impurity in spherical quantum dot with parabolic potential, Superlattice Microst. 60, 389-397 (2013).

[18] L. Máthé, C. P. Onyenegecha, A. -A. Farcaş, L. -M. Pioraş-Ţimbolmaş, M. Solaimani and H. Hassanabadi, Linear and nonlinear optical properties in spherical quantum dots: Inversely quadratic Hellmann potential, Phys. Lett. 397, 127262 (2021).

[19] A. Naifar, N. Zeiri, S. Abdi-Ben Nasrallah and M. Said, Linear and nonlinear optical properties of CdSe/ZnTe core/shell spherical quantum dots embedded in different dielectric matrices, Photonics and Nanostructures 40, 100789 (2020).

[20] E. B. Al, E. Kasapoglu, S. Sakiroglu, H. Sari, I. Sökmen and C. A. Duque, Binding energies and optical absorption of donor impurities in spherical quantum dot under applied magnetic field, Physica E 119, 114011 (2020).

[21] D. Dorfs, S. Hickey and A. Eychmüller, Type-I and Type-II core-shell quantum dots: Synthesis and characterization, Kumar CSSR (Ed) Semiconductor Nanomaterials, Weinheim: Wiley-VCH, 331-366 (2010).

[22] M. V. Maximov, L. V. Asryan, Yu. M. Shernyakov, A. F. Tsatsul'nikov, I. N. Kaiander, V. V. Nikolaev, A. R. Kovsh, S. S. Mikhrin, V. M. Ustinov, A. E. Zhukov, Zh. I. Alferov, N. N. Ledenstov and D. Bimberg, Gain and threshold characteristics of long wavelength lasers based on InAs/GaAs quantum dots formed by activated alloy phase separation, IEEE J. Quant. Electron. 37, 676-683 (2001).

[23] A. Chafai, I. Essaoudi, A. Ainane and R. Ahuja, Linear and nonlinear optical properties of donors inside a CdSe/ZnTe core/shell nanodot: Role of size modulation, Results Phys. 14, 102414 (2019).

[24] S. M'zerd, M. El Haouari, A. Talbi, E. Feddi and M. E. Mora-Ramos, Impact of electron-LO-phonon correction and donor impurity localization on the linear and nonlinear optical properties in spherical core/shell semiconductor quantum dots, J. Alloy. Comp. 753, 68-78 (2018).

[25] E. Feddi, A. Talbi, M. E. Mora-Ramos, M. El Haouari, F. Dujardin and C. A. Duque, Linear and nonlinear magneto-optical properties of an off-center single dopant in a spherical core/shell quantum dot, Physica B 524, 64-70 (2017).

[26] M. El Haouari, A. Talbi, E. Feddi, H. El Ghazi, A. Oukerroum and F. Dujardin, Linear and nonlinear optical properties 
of a single dopant in strained AlAs/GaAs spherical core/shell quantum dots, Optic. Comm. 383, 231-237 (2017).

[27] Zhi-Hai Zhang, G. Zhuang, Kang-Xian Guo and Jian-Hui Yuan, Donor-impurity-related optical absorption and refractive index changes in GaAs/AlGaAs core/shell spherical quantum dots, Superlattice Microst. 100, $440-447$ (2016).

[28] P. C. L. Jasmine, A. J. Peter and C. W. Lee, Intersubband optical transition energy in a CdTe/Zn $n_{0.2} C d 0.8 T e / Z n T e$ core/shell/shell spherical quantum dot with Smorodinsky-Winternitz confinement potential, Chem. Phys. 452, 40-45 (2015).

[29] S. Adachi, GaAs, AlAs, and $A l_{x} G a_{1-x} A s$ : Material parameters for use in research and device applications, J. Appl. Phys. 58, R1-R29 (1985).

[30] V. A. Holovatsky, I. B. Bernik and M. Ya. Yakhnevych, Effect of magnetic field on energy spectrum and localization of electron in $\mathrm{CdS} / \mathrm{HgS} / \mathrm{CdS} / \mathrm{HgS} / \mathrm{CdS}$ multilayered spherical nanostructure, Physica B 508, 112-117 (2017).

[31] V. Holovatsky, O. Voitsekhivska and I. Bernik, Effect of magnetic field on electron spectrum in spherical nano-structures, Condens. Matter. Phys. 17, 13702 (2014).

[32] V. A. Holovatsky, O. M. Voitsekhivska and M. Ya Yakhnevych, The effect of magnetic field and donor impurity on electron spectrum in spherical core-shell quantum dot, Superlattice Microst. 116, 9-16 (2018). 